Anales de Geografía de la Universidad Complutense ISSN: 0211-9803

http://dx.doi.org/10.5209/AGUC.60476

\title{
Comercio minorista y peatonalización: evolución y adaptación en la ciudad costera de Malgrat de Mar (Barcelona)
}

\author{
Meritxell Simó López ${ }^{1}$; Antònia Casellas ${ }^{2}$; Pau Avellaneda ${ }^{3}$ \\ Recibido: 7 de octubre del 2016 / Enviado a evaluar: 29 de enero de 12017/ Aceptado: 14 de diciembre del 2017
}

\begin{abstract}
Resumen. La desaparición del comercio minorista del centro urbano es uno de los principales problemas que afecta a las ciudades del siglo XXI. El presente trabajo analiza la evolución de este tipo de comercio en zonas comerciales centrales vinculándolo a los efectos de la peatonalización y al cambio de ciclo económico en Malgrat de Mar, una ciudad costera de la provincia de Barcelona. Con una metodología cualitativa basada en entrevistas semi-estructuradas y el análisis de datos socio-económicos y documentos de planificación urbanística se estudian los factores que influyen en el cierre de establecimientos y el cambio de actividades comerciales durante el período 2007-2015. Los resultados indican que, contrariamente a la opinión generalizada de la población local, la rehabilitación urbanística y la estrategia de peatonalización impulsada por el Ayuntamiento han contribuido a mantener la viabilidad de los comercios minoristas. Otros factores positivos identificados son la adaptación y especialización de los comercios a los nuevos hábitos de consumo.
\end{abstract}

Palabras clave: Peatonalización; comercio minorista; dinamización económica; ciudades costeras.

\section{[en] Retail shops and pedestrian areas: evolution and adaptation in the coastal city of Malgrat de Mar (Barcelona)}

\footnotetext{
1 Departamento de Geografía. Universidad Autónoma de Barcelona.

E-mail: msimolop@gmail.com

2 Departamento de Geografía. Universidad Autónoma de Barcelona.

E-mail: antonia.casellas@uab.cat

3 Departamento de Geografía. Universidad Autónoma de Barcelona.

E-mail: pau.avellaneda@uab.cat
}

Abstract. The disappearance of traditional downtown shops is one of the main problems affecting urban areas in the twenty-first century. This paper analyzes the evolution of mom and pop stores linking it to the effects of implementing new pedestrian paths and changes in the economic cycle in Malgrat de Mar, a coastal city in Barcelona province. With a qualitative methodology based on semi-structured interviews and the analysis of socio-economic data and urban planning documents, the paper analyses the factors influencing the closure of stores and the changes on business during the period 2007-2015. The results 
indicate that, contrary to the local population's general opinion, urban rehabilitation and pedestrian strategies driven by the City Council have helped to maintain the viability of retail. Other positive factors include the adaptation and specialization of businesses to new consumer preferences.

Keywords: Pedestrian areas; downtown retail; economic revitalization; coastal cities.

\section{[fr] Commerce de détail et piétonisation: évolution et adaptation dans la ville côtière de Malgrat de Mar (Barcelone)}

Résumé. La disparition du commerce de détail du centre urbain est l'un des principaux problèmes qui affectent les villes du 21 ème siècle. Cet article analyse l'évolution de ce commerce dans les principaux secteurs d'activité reliant les effets de la piétonisation et l'évolution du cycle économique à Malgrat de Mar, une ville côtière dans la province de Barcelone. Avec une méthodologie qualitative basée sur des entretiens semi-structurés et analyse des données socio-économiques et les documents des facteurs de planification urbaine qui influent sur la fermeture et l'évolution des activités au cours de la période 20072015 , ils sont étudiés. Les résultats indiquent que, contrairement à une opinion répandue de la population locale, la réhabilitation urbaine et de la stratégie de peatonalización entraînée par le Conseil municipal ont contribué à maintenir la viabilité des points de vente. Les autres facteurs positifs identifiés sont l'adaptation et la spécialisation des magasins aux nouvelles habitudes de consommation.

Mots clés: Piétonisation; commerce de détail; revitalisation économique; villes côtières.

Cómo citar. Simó López, M., Casellas, A. y Avellaneda, P. (2018): Comercio minorista y peatonalización: evolución y adaptación en la ciudad costera de Malgrat de Mar (Barcelona). Anales de Geografia de la Universidad Complutense, 38(1), 219-238.

Sumario. 1. Introducción. 2. El comercio minorista y la peatonalización. 3. Ciudad costera, comercio minorista y peatonalización del centro histórico de Malgrat de Mar. 4. La actividad comercial en contexto. 4.1. Las grandes superficies y supermercados en Malgrat de Mar y sus alrededores como competencia. 4.2. La peatonalización y la evolución de la actividad comercial. 5. Percepciones acerca de la peatonalización y respuestas del comercio local a la crisis del comercio minorista. 6. Reflexiones finales. 7. Bibliografía.

\section{Introducción}

En contraposición con la experiencia de países anglosajones en los que las tradicionales tiendas del centro urbano empezaron a decaer en la década de los años 1960 como consecuencia de la consolidación del proceso de suburbanización, en España el modelo comercial minorista en muchas ciudades se mantuvo sin grandes dificultades hasta mediados de los años 1980. Sin embargo, a partir de este momento, este tipo de comercio entra paulatinamente en un proceso de lucha desigual para no desaparecer. Los cambios en los hábitos de consumo, la aparición de las grandes superficies comerciales en el extrarradio y su gran aceptación por parte de los consumidores, la falta de relevo generacional, la apertura de tiendas sin un riguroso estudio de mercado y la universalización del uso del coche y de Internet son algunos de los factores que han influido de forma creciente en el declive de la actividad comercial del centro de la ciudad, lo cual se ha visto reflejado a través del cierre de 
comercios minoristas en los centros urbanos (Cuesta, 1999; Gómez, 2000; Herrera, 2012; Matás, 2013; Somoza-Medina y López-González, 2017).

En un intento de dar respuestas a los retos del comercio local, desde el año 1996 en España se han impulsado numerosos proyectos destinados a fomentar las asociaciones para mejorar la competitividad del pequeño y mediano comercio (Conride, 2003). Algunas de las estrategias de las asociaciones de comerciantes se han centrado en acciones puntuales para la mejora del espacio público, y en la creación de redes de cooperación entre los distintos comercios, agentes locales y la administración pública con el fin de mejorar el atractivo de la actividad comercial (Gómez, 2000; Baringo y De Gavira, 2001). En el caso de Cataluña, en el análisis de la evolución de los comercios tradicionales se constata que a partir de los años 1960, en un periodo de crecimiento económico y expansión de la clase media, los comercios familiares de las grandes ciudades amplían sus locales y se modernizan. Así mismo, y en respuesta a estos cambios económicos y sociales, durante los años 1970 surgen los primeros supermercados que entran en competición con el comercio minorista, que en algunos casos empieza a ser deficitario. Durante los años 1980 el proceso se agudiza con la instalación de las primeras franquicias, a la vez que prosigue la apertura de supermercados en el interior de las poblaciones y aparecen los primeros supermercados de mayor tamaño en la periferia (Espinosa Seguí, 2003).

Durante las décadas de 1980 y 1990, y en respuesta a los cambios apreciados en la estructura comercial y residencial, los gobiernos municipales emprenden acciones de recuperación y rehabilitación de barrios degradados (Batallé, 2000), con un creciente énfasis en la creación de imagen (Casellas, Dot, Pallares-Barbera, 2010; Casellas, 2016). Durante este período la Generalitat de Catalunya financia reformas de locales comerciales, mercados municipales y creación de islas peatonales. La creación de zonas peatonales para formar un centro comercial urbano atractivo significa un paso importante en la modernización del comercio minorista (Herrera, 2012), que no siempre es bien acogido por los comerciantes (Bergua, 2002).

$\mathrm{Si}$ bien las dificultades del pequeño comercio en ciudades grandes han sido analizadas desde distintas perspectivas, vinculándolo a los efectos de los supermercados (Borraz, et al. 2014) y las dinámicas del ámbito metropolitano (Franklin, 2001), en un contexto de presión generalizada hacia el comercio local minorista los retos del comercio en las ciudades de tamaño medio y pequeño cuentan con un menor número de análisis pormenorizados. El presente trabajo aporta información sobre este ámbito al centrarse en la evolución del comercio en el municipio costero de Malgrat de Mar (Barcelona), una ciudad turística de poco más de 18.000 habitantes. El trabajo se estructura a partir de dos objetivos. En primer lugar se analizan los factores que han influido en el cierre de actividades comerciales en el centro del municipio de Malgrat de Mar a lo largo del período 2007-15; y en segundo lugar se evalúa qué papel ha jugado la peatonalización en este proceso, ya que a priori amplias capas de la población local y agentes comerciales han asociado la peatonalización y la política de pacificación del tráfico en la ciudad a la pérdida de actividad comercial. En este sentido, el caso de Malgrat de Mar aporta datos que se pueden extrapolar a otros casos con características comerciales y socio-demográficas 
similares. El objetivo del trabajo responde a la voluntad de contribuir al debate académico sobre las dinámicas de la peatonalización y el comercio minorista, y aportar datos para informar políticas urbanas en ciudades medias y pequeñas. Para ello, en los siguientes apartados se discuten los factores que afectan a la viabilidad del comercio minorista para después centrarnos en el análisis del caso de estudio.

\section{El comercio minorista y la peatonalización}

El comercio minorista equilibra y estructura la trama urbana pero también contribuye a darle personalidad, imagen y vitalidad. La pérdida de actividad comercial es uno de los indicadores más significativos de la decadencia de los centros urbanos. Cornide (2003) enfatiza la importancia que tiene el comercio minorista en las ciudades al apuntar que:

"El comercio es esencial en la ciudad porque 'no existe ciudad sin comercio'. El comercio tradicional da identidad pero aparte es un estilo de vida, de cultura y de historia que a la vez da al vecino sentido de pertenencia y fortalece el tejido urbano y la trama social de la ciudad. Cuando el centro comercial se degrada sobreviene la inseguridad. Cierran comercios, se van vecinos, decaen servicios, desaparece el trabajo, la ciudad de desertiza"

(Conride, 2003 p.3).

Uno de los principales factores que se han identificado como elementos que intervienen en la viabilidad del comercio minorista es la peatonalización. La peatonalización se puede definir como el fenómeno asociado al cierre total o parcial de las calles al tráfico motorizado privado. Su objetivo es crear espacios exclusivos para los viandantes a partir de vías anteriormente destinadas a todo tipo de vehículos. El objetivo principal de la peatonalización es pues crear condiciones favorables para que el paseo resulte agradable para residentes y visitantes. Como apunta Jane Jacobs "El lugar público principal de una ciudad son las calles y las aceras, son sus órganos más vitales. "Cuándo pensamos en una ciudad, ¿que nos viene a la mente? Sus calles. Si las calles de una ciudad se ven interesantes, la ciudad se ve interesante, y si se ven aburridos, la ciudad se ve opaca" (Jacobs, 1961, p.29).

Contrariamente a lo que pudiera parecer, la peatonalización no es un fenómeno reciente. Esta política urbanística de mejora del entorno físico con el fin de dinamizar los centros urbanos se remonta a los años 1920 en ciudades de los Estados Unidos. En ciudades europeas aparece posteriormente, y lo hace como forma de rehabilitación urbanística de los centros históricos con el fin de liberar los espacios de circulación rodada y recuperar el espacio para el peatón. El objetivo es mejorar la cohesión social, la habitabilidad y la calidad de vida. Las ciudades de Essen y Colonia en Alemania y Copenhague en Dinamarca destacan como ciudades europeas pioneras en peatonalización (Del Campo, García \& Flores, 2009). Esta estrategia se expande a partir de la Cumbre de la Tierra de Río de Janeiro de 1992, cuando, en el marco generado por los principios del desarrollo sostenible, se impulsan multitud de 
iniciativas locales de peatonalización en distintas ciudades europeas. La finalidad de esta estrategia de peatonalización urbana en centros comerciales urbanos es reducir la contaminación acústica, mejorar la seguridad vial y potenciar el desarrollo económico a través de los centros comerciales abiertos (Campesinos, 1990; Sanz, 1996; González, 2006).

Numerosas experiencias aportan evidencias que demuestran como la creación de las islas peatonales en los centros de la ciudad pueden funcionar como herramienta de dinamización comercial generando un mayor atractivo comercial, social y económico (Gómez, 2000). A su vez, si se produce una buena aplicación en la creación de calles peatonales, éstas pueden convertirse en un recurso turístico y de ocio (Troitiño, 2003). Éste es el caso, por ejemplo, de Sant Cugat del Vallès, una ciudad de poco menos de 89.000 habitantes en 2016, ubicada dentro del área metropolitana de Barcelona. La peatonalización de una de las principales calles del municipio generó inicialmente reticencias, pero demostró a la larga la creación de oportunidades de negocio a los comercios que ya existían en la zona, al mismo tiempo que se convirtió en un espacio tractor de otros negocios, especialmente del ámbito de la restauración, debido al efecto llamada. Una encuesta realizada en 2014 con el fin de valorar los efectos de la peatonalización concluyó que el $55 \%$ de los comercios consideraban que la implantación de la zona peatonal había influido en la mejora de su negocio; a su vez, un $73 \%$ de los comercios instalados después de la peatonalización afirmaban que ésta había tenido una influencia decisiva en la implantación de su negocio en este espacio (Ayuntamiento de Sant Cugat del Vallès, 2014).

En algunas ciudades pequeñas y medias de la región de Barcelona la peatonalización también se ha identificado como factor que ha contribuido a atenuar los efectos de la crisis económica. A diferencia de otros espacios no peatonalizados, hay evidencias que demuestran que donde se han realizado este tipo de intervención los estragos de la crisis han sido menores en tanto que estos espacios han seguido siendo zonas más activas comercialmente que el resto de áreas comerciales abiertas al tráfico. Un ejemplo se observa en el municipio de Granollers. En 2013, con un censo de 2.434 actividades comerciales en activo en planta baja, la ciudad de Granollers tenía un total de 780 locales vacíos. En sus barrios céntricos comerciales el porcentaje de locales vacíos era del $17 \%$, una proporción similar a otras capitales comerciales de la región. Sin embargo, en la zona peatonal la ocupación de locales comerciales alcanzaba el $97 \%$, siendo sólo de un 3\% los locales vacíos (Ayuntamiento de Granollers, 2013).

En el análisis de los beneficios de la peatonalización cabe señalar sin embargo que la mera implantación de áreas peatonales no es suficiente para dinamizar o revitalizar el comercio minorista tradicional. Para que ello ocurra, el análisis de experiencias exitosas demuestra que es necesario complementar esta iniciativa con otras políticas. De entre ellas destaca: 1) facilitar el acceso a la zona, especialmente a los no residentes, 2) adaptar los comercios a un mercado donde los hábitos de consumo y los gustos de los consumidores evolucionan constantemente, 3) diferenciarse del resto de comercios ofreciendo servicios personalizados, tarjetas de fidelización, campañas publicitarias, y 4) capitalizar las posibilidades que ofrecen las nuevas tecnologías, 
como por ejemplo la compra por internet (Gómez, 2000; Varisco, 2005; SomozaMedina y López-González, 2017). Un caso paradigmático en este sentido es la ciudad de Pontevedra donde, paralelamente a una importante intervención de renovación urbana consistente en la peatonalización de los espacios centrales, se han emprendido acciones para conservar el comercio tradicional e impedir la deslocalización comercial, ofreciendo un centro comercial atractivo, a la vez que se ha implementado una política de no concesión de licencias para grandes superficies comerciales dentro del casco urbano de la ciudad (Concello de Pontevedra, 2011).

Finalmente es necesario remarcar que la peatonalización no está exenta de problemáticas. La transformación de una calle comercial en peatonal puede resultar polémica y rechazada, como sucede a menudo en un primer momento, por los comerciantes e incluso los residentes que perciben una restricción al tráfico rodado que puede perjudicarles en su actividad económica o en sus desplazamientos por razones de trabajo $u$ ocio. Por tanto, antes de implementar políticas de peatonalización y restricción del tráfico se requiere un estudio preliminar para analizar el posible impacto de estas medidas y la inclusión de diferentes colectivos en procesos participativos. Los estudios empíricos señalan que es importante tener la aprobación de los comerciantes ya que la peatonalización puede tener consecuencias positivas pero también, en determinados casos, contraproducentes (Varisco, 2005). Así mismo, los efectos negativos de la peatonalización pueden relacionarse con procesos de gentrificación a través del aumento del precio del suelo que consecuentemente deriva en expulsión de usos residenciales y cierre y substitución de comercios tradicionales por oficinas y establecimientos más especializados. Este proceso puede además ir acompañado de la comodificación y privatización del espacio público, a través del uso de aceras o paseos para actividades comerciales como las asociadas a las terrazas de bares y restaurantes (Sanz, 1996, Atkinson, 2003; Zukin 1995 y 1998).

\section{Ciudad costera, comercio minorista y peatonalización del centro histórico de Malgrat de Mar}

Malgrat de Mar es un municipio costero situado en el extremo norte oriental de la provincia de Barcelona, con una superficie de $8,8 \mathrm{~km}^{2}$ y una población de 18.295 habitantes en el 2016 (Institut d'Estadística de Catalunya [IDESCAT], 2017) (Figura 1). Dentro del municipio, el área de análisis de esta investigación se centra en la zona del centro histórico del municipio, en concreto, la zona central peatonal donde se concentra la actividad comercial, en la que se distinguen dos espacios diferenciados. El primero pertenece a la primera peatonalización del centro realizada en el periodo 2000-2004, calles del Mar y Ramón Turró, y que conecta el frente marítimo con el centro urbano. En esta zona se concentra el comercio minorista de la ciudad. El segundo corresponde a la peatonalización realizada en el período 2009-2010 de la zona central del municipio, una zona donde predomina un uso más residencial combinado con el comercio minorista y los servicios (Figura 2). 
Figura 1. Malgrat de Mar dentro de la Región de Barcelona.

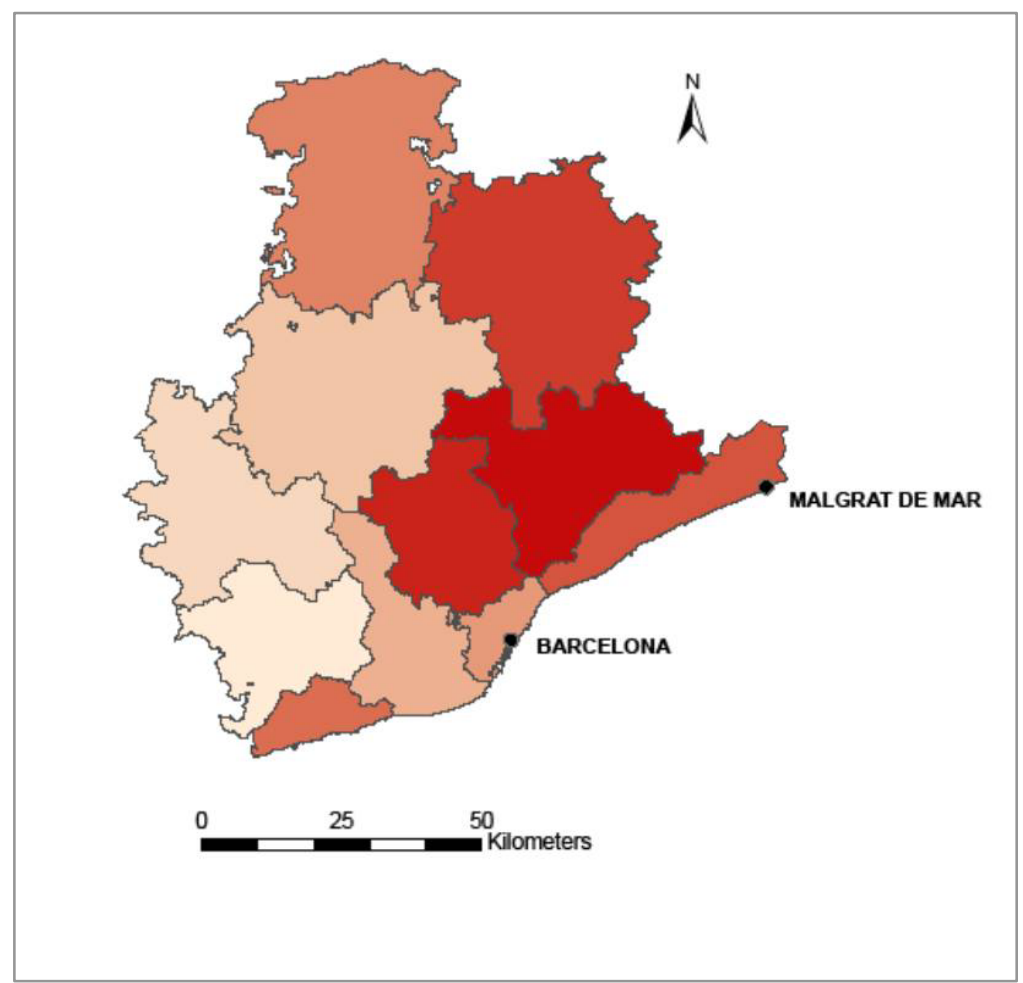

Fuente: Elaboración propia a partir de datos extraídos del Institut Cartogràfic de Catalunya [ICC]

La economía del municipio de Malgrat de Mar tiene su base principal en el sector terciario (73\% de la población ocupada) (IDESCAT, 2011). Si bien el componente comercial estuvo siempre presente en la base económica del municipio, a partir de la década de los años 1990, la diversificación productiva permitió la especialización de los comercios. De esta forma, la estructura comercial se fundamenta en la progresiva modernización de los establecimientos para adecuarse a los patrones de consumo de un municipio costero y turístico. Siguiendo una dinámica general al resto del área metropolitana de Barcelona, en Malgrat de Mar a partir de los años 1980 se produce la apertura de grandes superficies comerciales y supermercados fuera del casco urbano, situación que deriva en un aumento de la competencia y la pérdida de atractivo del comercio minorista (Valbonesi, 2005). A partir del año 2008, como ocurrió de forma generalizada en España, la crisis económica perjudicó al sector comercial produciéndose el cierre de comercios en el centro de la ciudad (Ayuntamiento Malgrat de Mar, 2015).

Con relación a las pautas demográficas y los usos del suelo, junto con el constante crecimiento del municipio y la segregación de usos en el territorio, los desplazamientos internos por trabajo y estudio en modos motorizados han ido en 
aumento en las últimas décadas. Ya en el 2001, la mayor parte $(51 \%)$ de desplazamientos se realizaban en vehículos privados (45\% coche y $6 \%$ moto), $40 \%$ a pie y 7\% en bicicleta (Encuesta de Movilidad Obligada [EMO], 2001). Este dato ejemplifica un hábito adquirido entre la población de dependencia del vehículo privado que condiciona el nivel de la aceptación de los proyectos de peatonalización de las calles del centro del municipio.

En los siguientes apartados, el estudio se centra en identificar cuáles son los factores que influyen en el cierre de los establecimientos comerciales en la zona central peatonal de Malgrat de Mar en el período 2007-2015. Para contextualizar el análisis se ha realizado una búsqueda documental de estudios referentes al comercio en el municipio, dedicando especial atención a la información procedente del Programa de Orientación para los Equipamientos Comerciales (POEC), realizado con datos del 2006 y aprobado en junio del 2009. Con el fin de complementar la información se han recopilado datos del departamento de urbanismo del municipio acerca de la evolución de las distintas actividades comerciales que operan en la zona central de la población. Finalmente, a través de la tasa de residuos se ha obtenido el número de locales cerrados registrados en mayo del 2015, lo cual ha permitido realizar un análisis comparativo entre los años 2007 y 2015.

El trabajo de campo también ha requerido la observación directa de la zona de estudio. En la primera fase se identificaron el número de locales abiertos y cerrados en las calles de la primera y segunda peatonalización. Estos datos permitieron comparar los obtenidos en marzo de 2015 con los datos recogidos por el POEC el año 2007. En la siguiente fase del trabajo de campo se clasificó el comercio siguiendo la clasificación de actividades económicas (CCAE 2009). El trabajo de observación permitió poder determinar cambios en el número de establecimientos abiertos durante el período de verano, un periodo importante para el análisis al tratarse de una ciudad costera en la que la estacionalidad vinculada al turismo influye en las pautas comerciales. El trabajo se complementó con entrevistas semi-estructuradas. Se realizaron un total de 13 entrevistas semi-estructuradas: 3 a representantes institucionales y 10 a comerciantes. Las entrevistas permiten obtener información sobre la percepción de diferentes colectivos acerca de la problemática del cierre de los comercios, la peatonalización y las iniciativas promovidas con el fin de revitalizar la zona comercial por parte del Ayuntamiento y de la Asociación de Comerciantes.

\section{La actividad comercial en contexto}

\subsection{Las grandes superficies y supermercados en Malgrat de Mar y sus alrededores como competencia}

Los locales comerciales tradicionales concentrados en el centro de Malgrat de Mar no superan los $100 \mathrm{~m}^{2}$ y acostumbran a ser tiendas especializadas en productos personales y del hogar. Tal y como se ha indicado anteriormente, una de las causas más frecuentes en el cierre de estos comercios es su proximidad a centros comerciales o grandes superficies con oferta mucho más amplia. Los hábitos de consumo han 
variado y la población busca comodidad, proximidad, ahorro y espacios de ocio, entre otros factores. En la Tabla 1 se presentan las características de los seis centros comerciales más cercanos al municipio de Malgrat de Mar con posibilidad de aparcamiento gratuito. Como puede observarse, un centro comercial con un número significativo de tiendas y oferta a $3 \mathrm{~km}$ del centro de Malgrat de Mar y el resto de centros distan del municipio entre $37 \mathrm{~km}$ a $63 \mathrm{~km}$.

Tabla 1. Centros comerciales cercanos a Malgrat de Mar (2015)

\begin{tabular}{|l|c|c|c|c|c|c|}
\hline $\begin{array}{l}\text { Centro } \\
\text { comercial }\end{array}$ & Municipio & $\begin{array}{c}\text { Año } \\
\text { apertura }\end{array}$ & $\begin{array}{c}\text { Superficie } \\
\text { bruta } \\
\text { alquilable } \\
\text { (m2) }\end{array}$ & $\mathbf{N}^{\circ}$ tiendas & $\begin{array}{c}\text { Plazas de } \\
\text { aparcamiento }\end{array}$ & $\begin{array}{c}\text { Distancia de } \\
\text { Malgrat de } \\
\text { Mar } \\
\text { (km) }\end{array}$ \\
\hline Susanna & Santa Susanna & 1993 & 9.000 & 14 & 600 & 3 \\
\hline Mataró Park & Mataró & 2000 & 62.000 & 156 & 3.200 & 37 \\
\hline Espai Gironès & Salt & 2005 & 46.000 & 122 & 2.200 & 42 \\
\hline Montigalà & Badalona & 1991 & 35.545 & 61 & 2.618 & 58 \\
\hline Diagonal Mar & Barcelona & 2001 & 87.085 & 220 & 5.000 & 63 \\
\hline La Maquinista & Barcelona & 2001 & 77.500 & 227 & 4.800 & 63 \\
\hline
\end{tabular}

Fuente: Elaboración propia a partir de datos extraídos del informe POEC y Google Maps (distancia).

Otro grupo de comercios que podrían entrar en competencia con el comercio minorista asentado en el centro histórico de Malgrat de Mar y, especialmente, con el ubicado en el área de estudio, son los supermercados. Se trata de comercios dedicados sobre todo a la alimentación y productos para el hogar con una superficie mayor que la del comercio minorista.

Tabla 2. Supermercados Malgrat de Mar (2015).

\begin{tabular}{|r|c|r|}
\hline Año ape rtura & Supermercado & \multicolumn{1}{|c|}{$\begin{array}{c}\text { Superficie } \\
\left(\mathbf{m}^{\mathbf{2}}\right)\end{array}$} \\
\hline 1982 & Próxim & 162 \\
\hline 1990 & Spar & 75 \\
\hline 1997 & Caprabo & 1654 \\
\hline 2002 & Dia & 429 \\
\hline 2002 & Aldi & 847 \\
\hline 2006 & Suma & 355 \\
\hline 2010 & Mercadona & 5862 \\
\hline 2012 & Happy minimarket & 21,3 \\
\hline 2013 & Distop & 176 \\
\hline
\end{tabular}

Fuente: Elaboración propia a partir de datos extraídos del Ayuntamiento de Malgrat de Mar. 
El análisis del año de apertura y la superficie de los supermercados existentes en Malgrat de Mar (Tabla 2) muestra que hay dos supermercados que se sitúan en la parte central del municipio y que no ofrecen plazas de aparcamiento. El resto de supermercados se encuentran ubicados en la periferia de la población. La información obtenida permite observar que a partir de la década del 2000, en Malgrat de Mar se amplía considerablemente tanto el número de establecimientos como la superficie de éstos, siendo el año 2010 un punto de inflexión significativo con la apertura de un supermercado altamente competitivo, tanto por sus dimensiones como por su política de precios y ofertas (Tabla 2).

\subsection{La peatonalización y la evolución de la actividad comercial}

Como enfatizan numerosos estudios, la monopolización del espacio público por parte del vehículo a motor, ya sea estacionado o en circulación, especialmente en las calles estrechas de los centros históricos, generan dificultades de accesibilidad y movilidad peatonal, desincentivado la presencia y la permanencia de los ciudadanos en estos espacios y, en consecuencia, deteriorando la actividad comercial. Desde esta perspectiva, y con el objetivo de mejorar la calidad urbana del espacio central, en Malgrat de Mar a lo largo de los años se realizaron mejoras urbanísticas y de accesibilidad para mejorar y potenciar el comercio minorista del centro. En 1998 se aprobó el primer Plan de Movilidad que pretendía recuperar el espacio público para el uso social y cultural y, en consecuencia, mejorar la calidad de vida de los residentes en Malgrat de Mar. Para ello se limitó la velocidad de circulación dentro del municipio, creando islas peatonales y aparcamientos.

A partir de este punto de inflexión, el proceso de peatonalización del centro de la ciudad se ha desarrollado en diferentes fases. Durante el periodo 2000-2004 se implementó la primera peatonalización del municipio en las calles Mar y Ramón Turró. En 2005 se hizo un Plan de Accesibilidad que propuso alternativas para extender la pacificación del tráfico a otras calles del municipio. En esta misma línea, en el año 2007 se presentó un nuevo Plan Local de Seguridad Vial con el objetivo de disminuir un $25 \%$ la accidentalidad dentro del municipio. Finalmente, en el año 2009 se aprobó definitivamente el Proyecto de Renovación Urbana del núcleo antiguo en el cual se realizó una actuación integral para dinamizar la actividad turística y comercial vinculándola a la peatonalización. En líneas generales, los objetivos propuestos en este último proyecto para la ciudad se orientaban a reordenar el espacio urbano para incrementar el atractivo turístico, potenciar el uso comercial a través de las propuestas del POEC, homogeneizar pavimentos, mejorar el mobiliario urbano, el alcantarillado, la gestión de aguas, ampliar las plazas de aparcamiento del centro y mejorar la red viaria siguiendo las directrices del Plan de Movilidad. Bajo estas directrices se llevó a cabo la segunda peatonalización en la ciudad en 2009-2010. 
Figura 2. Ortofoto del municipio y área de estudio.

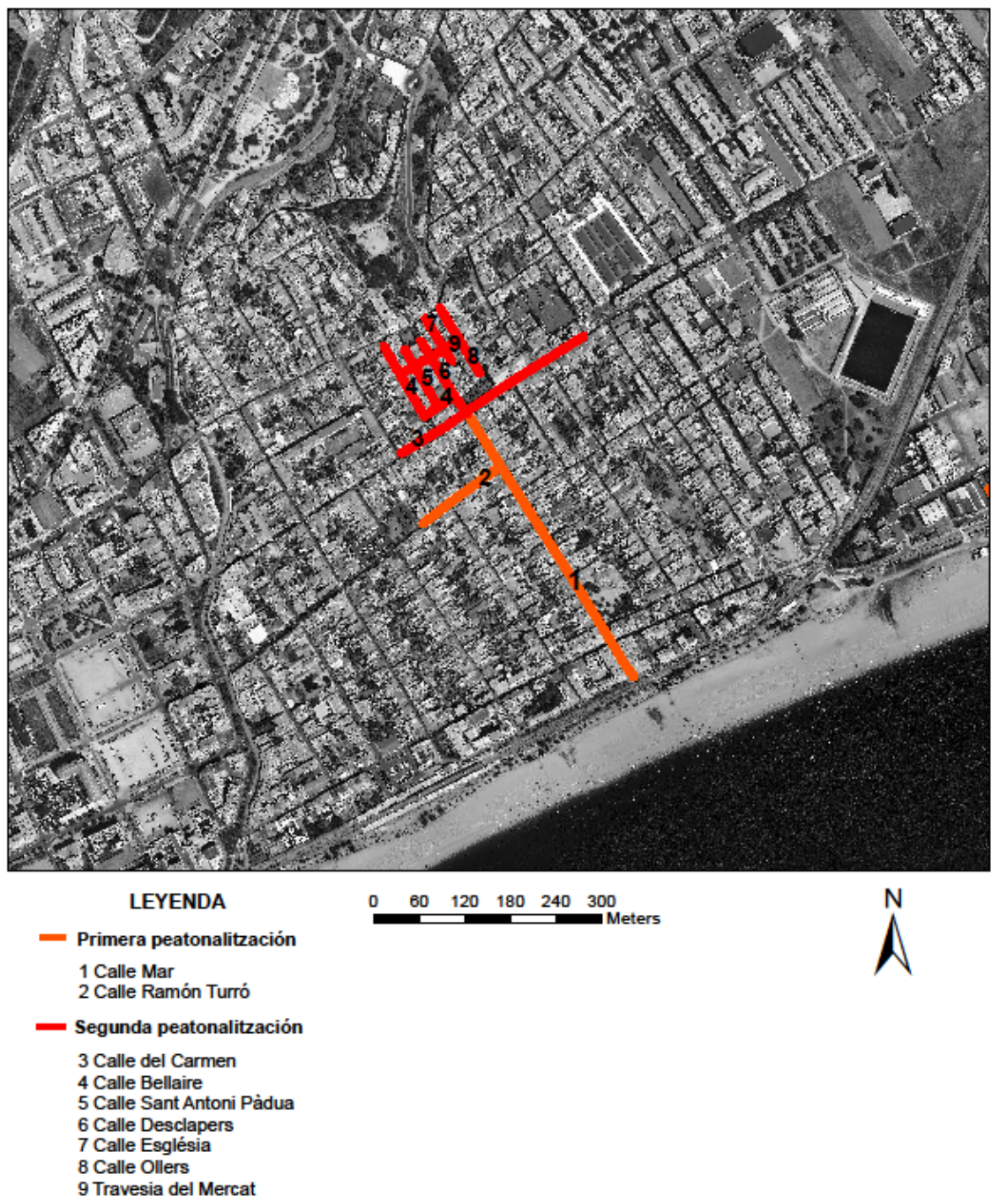

Fuente: Elaboración propia.

Los efectos de la crisis económica iniciada en el 2008 y cuyos efectos empezaron a evidenciarse a partir del 2009, coincidieron con la segunda peatonalización de la zona central que se produjo simultáneamente con el cierre de comercios en la ciudad. El análisis de los datos revela sin embargo que el porcentaje de locales cerrados en las calles de la zona central peatonal afectó de forma distinta a las diferentes calles analizadas. Variables como el período de peatonalización, la especialización 
comercial, la continuidad comercial y la existencia de viviendas en planta baja ayudan a explicar estas diferencias.

De una tasa de ocupación en 2007 del $92,3 \%$, con 24 comercios abiertos de un total de 26, la calle Ramón Turró pasó en 2015 a una ocupación del 85,2\%, con el cierre de 4 comercios. Esta calle destaca por ofrecer un comercio variado y especializado para el hogar y por un entorno menos comercial que otras calles del entorno debido al gran número de viviendas en planta baja, tal y como ya se evidenciaba en el análisis realizado por el POEC en el año 2007. En el 2015, sin embargo, y en pleno efecto de la crisis económica, el tramo peatonal evoluciona positivamente convirtiéndose en una de las calles donde se concentra un mayor dinamismo y actividad comercial. Por otro lado, la calle que tradicionalmente ha abarcado un mayor número de comercios, la calle del Mar, que comunica el centro de la ciudad con el frente marítimo, si en el año 2007 tenía 61 comercios abiertos de un total de 69 establecimientos, en el 2015 el número de locales comerciales había descendido a 68 , y de ellos sólo 50 (73,3\%) estaban en activo. La mitad norte de esta calle presentaba una imagen de concentración comercial y de servicios, mientras que la mitad sur mostraba baja concentración comercial producida por el aumento del número de locales cerrados, edificios deteriorados, presencia de equipamientos culturales y municipales, y una mayor concentración de viviendas y aparcamientos individuales. La elevada presencia de asesorías, bancos y equipamientos municipales conformaban un espacio con una marcada discontinuidad comercial (Figura 3).

Figura 3. Discontinuidad comercial en la calle del Mar (2015).

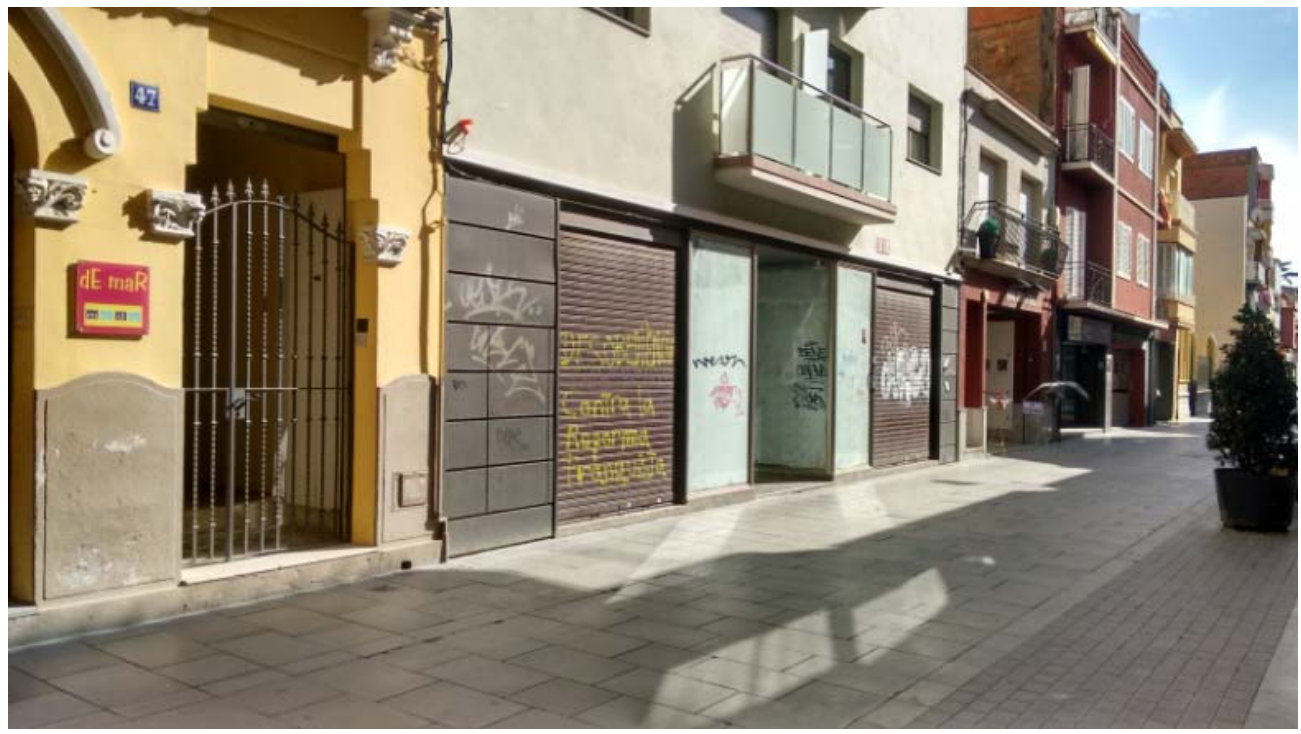

Fuente: Autores. 
A partir del análisis comparativo entre 2007 y 2015 se puede establecer que las calles del centro histórico que configuran la segunda peatonalización de la zona fueron las más afectadas por la crisis. La calle del Carme había sufrido una reducción significativa de tasa de ocupación de locales comerciales, del 7,5\% de locales cerrados en el 2007 se llegaba a $26,9 \%$ en el 2015. A través de la peatonalización, la zona adquirió un tejido comercial más compacto contribuyendo al aumento del atractivo del área, aunque en conjunto, se perdió un número significativo de comercio local. En general, la zona del casco antiguo con un gran potencial de atracción debido a la proximidad con las calles más comerciales y equipamientos culturales, no ha aumentado el número de comercios minoristas a pesar de haber incrementado el tráfico poblacional debido a la variedad de bares y restaurantes en la zona. En este sentido, el análisis de las actividades comerciales junto al proceso de peatonalización apunta a que ésta ha dinamizado el tráfico de visitantes, pero no ha ido acompañada de una mejora del comercio local. Una lectura de conjunto a partir del análisis de las cuatro áreas estudiadas nos permite señalar que las calles que han enfrentado con mayor éxito los retos generados por la crisis económica son aquellas que ya habían sido peatonalizadas con anterioridad al 2007. Las calles peatonalizadas una vez iniciada la crisis, por el contrario, son las que han sufrido un mayor porcentaje de cierres de locales comerciales lo que parecería evidenciar que la peatonalización por sí sola no es suficiente para revitalizar el comercio local.

Más allá de la peatonalización cabe señalar la especialización como un factor relevante para explicar los efectos de la crisis sobre el comercio local. Para analizar dicha variable se ha realizado un análisis clasificando las actividades económicas. Para ello se ha utilizado la Clasificación Catalana de Actividades Económicas (CCAE) del 2009, que se compone de 99 categorías y que identifica todas las actividades económicas industriales y de servicios. El grupo 47 pertenece al comercio minorista, el cual se define como comercio al detalle, excepto el comercio de vehículos a motor y motocicletas. Por otra parte, los servicios se clasifican con otra codificación más específica, que abarca del grupo 56 al 96, agrupando asesorías, bancos, clínicas dentales y academias de idiomas, entre otros.

En el conjunto de las tres calles de las que se dispone de datos comparativos entre 2007 y 2015 -Calle Ramón Turró, Calle del Mar y Calle del Carme- se ha producido un importante volumen de cierres de locales comerciales, pasando de 134 a 111, lo que supone una reducción del 17,2\% (Tabla 3). Sin embargo, es importante destacar que se aprecia una notable diferencia en la variación entre la respuesta del comercio minorista respecto al de los servicios. Así, si bien para el conjunto de las tres calles los locales dedicados a servicios han sufrido una reducción significativa del $33,8 \%$, el comercio al por menor apenas si han tenido una reducción del 1,4\%. 
Tabla 3. Variación comercios al por menor y servicios por calles (2007 y 2015)

\begin{tabular}{|l|c|c|c|c|}
\hline & \multicolumn{2}{|c|}{ Número de locales } & \multicolumn{2}{c|}{ Variación } \\
\cline { 2 - 5 } & 2007 & 2015 & $(\mathrm{abs})$ & $(\%)$ \\
\hline CALLE RAMON TURRÓ & 24 & 23 & -1 & $-4,2$ \\
\hline Comercio al por menor & 16 & 18 & 2 & 12,5 \\
\hline Servicios & 8 & 5 & -3 & $-37,5$ \\
\hline CALLE DE MAR & 61 & 50 & -11 & $-18,0$ \\
\hline Comercio al por menor & 33 & 29 & -4 & $-12,1$ \\
\hline Servicios & 28 & 21 & -7 & $-25,0$ \\
\hline CALLE DEL CARME & 49 & 38 & -11 & $-22,4$ \\
\hline Comercio al por menor & 20 & 21 & 1 & 5,0 \\
\hline Servicios & 29 & 17 & -12 & $-41,4$ \\
\hline TOTAL & 134 & 111 & -23 & $-17,2$ \\
\hline Comercio al por menor & 69 & 68 & -1 & $-1,4$ \\
\hline Servicios & 65 & 43 & -22 & $-33,8$ \\
\hline
\end{tabular}

Fuente: Elaboración propia a partir de datos extraídos del trabajo de campo, POEC, IDESCAT y el Ayuntamiento de Malgrat de Mar.

En conjunto, la reducción de la ocupación comercial se puede asociar al cierre de servicios como son oficinas bancarias, inmobiliarias y tiendas poco especializadas. A partir de los datos analizados se observa que el comercio minorista ha resistido mucho mejor que los servicios poco especializados. Esta es una variable que debería ser tomada en cuenta al establecer políticas de reactivación de áreas comerciales.

\section{Percepciones acerca de la peatonalización y respuestas del comercio local a la crisis del comercio minorista}

La segunda peatonalización del casco antiguo del municipio, realizada en 20092010, tenía como objetivo fomentar la movilidad peatonal libre del tráfico rodado. En su momento, el Ayuntamiento argumentó su iniciativa de peatonalización bajo criterios de mejoras urbanísticas y consolidación de la red comercial. Según los datos obtenidos a través de las entrevistas, en el año 2015, si bien esta segunda peatonalización no había conllevado mejoras en el comercio minorista debido a la multiplicidad de factores que influyen en el correcto funcionamiento de la zona y las distorsiones generadas por la crisis económica, desde el ámbito institucional se consideraba que había incrementado el número de personas que paseaban por el centro. 
"El comercio se ha visto afectado por el hecho de peatonalizar, la jubilación y la crisis económica, que fue el gran boom. Si queremos una explicación rápida decimos que peatonalizar no ha ido bien. Pero observando la situación real, vemos que han coincidido muchos factores y el peatonalizar ha servido como polo de atracción. No sabemos si sin peatonalizar sería tan atractivo pasear"

(Miembro Institucional 1).

Por otro lado, se apunta también a la importancia de tomar en cuenta las diferentes dinámicas de consumo en función de la franja horaria. Así, se señala que el centro de Malgrat de Mar:

"Funciona como centro de proximidad por las mañanas para realizar compras de primera necesidad, pero no como centro comercial activo. El comercio no tiene ningún atractivo a consecuencia de la falta de implicación por parte de los comerciantes, los cuales tendrían que apostar por calles iluminadas, escaparates destacados y adquirir una nueva consciencia"

(Miembro Institucional 2).

En este sentido, desde la administración también se apunta a que un factor dinamizador del comercio local es la especialización:

"Es cierto que han cerrado muchas tiendas por no afrontar como tenían que renovarse. Pero no todo es tan malo si todavía hay tiendas que funcionan. Los comercios parece que se ponen las pilas cambiando el producto $y$ especializándose para intentar captar a un nuevo consumidor",

(Miembro Institucional 1).

En esta misma línea de razonamiento, según los agentes institucionales, para mejorar la actividad comercial de la zona los comerciantes deberían adecuarse a los nuevos hábitos de consumo.

"La peatonalización puede ayudar a consolidar la red comercial pero también tenemos que analizar qué hacen los comerciantes para adecuarse a los nuevos cambios"

(Miembro Institucional, 1).

Del trabajo de campo realizado con miembros de colectivo institucional se desprende que el cierre de los comercios se percibe como resultado de una causalidad múltiple, destacando la falta de planificación por parte del comerciante, la competencia generada por la proximidad de las grandes superficies, la falta de relevo generacional al frente de negocios, que a menudo emplean sólo familiares que cotizan como trabajadores autónomos, y la falta de clientela por el cambio de hábitos de compra y consumo. 
La visión de los comerciantes, por el contrario, se centra en hacer énfasis en el cambio de las tendencias de consumo de la población y los efectos de la crisis económica, a la que se acusa de haber provocado la minimización de las ventas de productos que no son de primera necesidad. Otro de los factores negativos sobre los que la gran mayoría de comerciantes entrevistados coinciden es en señalar la falta de clientela joven. En respuesta a este diagnóstico, los comerciantes han tomado medidas de promoción a través de ofertas, alargar las rebajas, ofrecer horarios personalizados, realizar más cambios en los escaparates y modernizar el género adaptándolo a un público con un poder adquisitivo menor.

Respecto a la ubicación de los comercios y año de apertura, los comerciantes entrevistados ubicados en el centro con posterioridad al 2006 conservan la perspectiva de crecimiento económico y valoran estar ubicados en el centro peatonal. Enfatizan que para mantener sus establecimientos abiertos se han reinventado ofreciendo productos más económicos y en muchos casos on-line con distribución a domicilio.

"Cuando decidí poner mi negocio en la calle Ramón Turró intenté encontrar
género que me hiciera único. La diferencia con los otros comercios es que
tengo servicio de reparto a domicilio y a través de la página web he ampliado
mi negocio a otros municipios"

(Comerciante 9).

Cambios en las estrategias colectivas de los comerciantes han tenido como objetivo consolidar una red de comercio más compactada y fuerte relacionada con la estrategia de peatonalización. Con este fin, en 2004 se fusionaron las cuatro asociaciones de comerciantes del centro de la ciudad en una única asociación, la cual integró 135 comercios. A partir de las entrevistas realizadas se observa que el objetivo fundamental de la asociación fue peatonalizar la isla del casco antiguo con el fin de potenciarla como motor comercial. Según señalan los informantes, si bien las obras inicialmente fueron rechazadas por parte de algunos comerciantes y residentes por las dificultades causadas durante la ejecución y los cambios previstos para la eliminación del vehículo privado, las reformas se llevaron a cabo al entenderse que eran parte imprescindible del proyecto de reactivación comercial de la zona. Sin embargo, se critica la unilateralidad de la actuación municipal y la falta de participación de los comerciantes en el diseño de rehabilitación y peatonalización. Como indica el Comerciante 3,

"La administración no ha propuesto un debate de participación entre vecinos, consistorio y comerciantes para analizar los efectos que ha conllevado la rehabilitación, peatonalización y cambio de direcciones del casco antiguo que podría servir para encontrar soluciones y minimizar los errores"

(Comerciante 3).

Los comerciantes destacan, asimismo, que si bien la estrategia de la peatonalización es adecuada, la discontinuidad comercial en la Calle del Mar y los 
efectos de la crisis han influido negativamente en los comercios que todavía siguen abiertos.

"La crisis económica ha comportado una disminución de los individuos que frecuentan las tiendas para comprar y la peatonalización ha coincidido con este periodo"

(Comerciante 2).

Para intentar dinamizar el sector sur de la Calle del Mar y el resto de calles comerciales peatonalizadas, desde promoción económica y turística el Ayuntamiento ha propuesto acciones de dinamización alternativas como la apertura del comercio por la noche una vez al año, la opción para los comerciantes locales de tener una parada con ofertas el día de mercado ambulante, así como la promoción en ferias de invierno y primavera.

Figura 4. Feria de primavera, calle del Carme 2015.

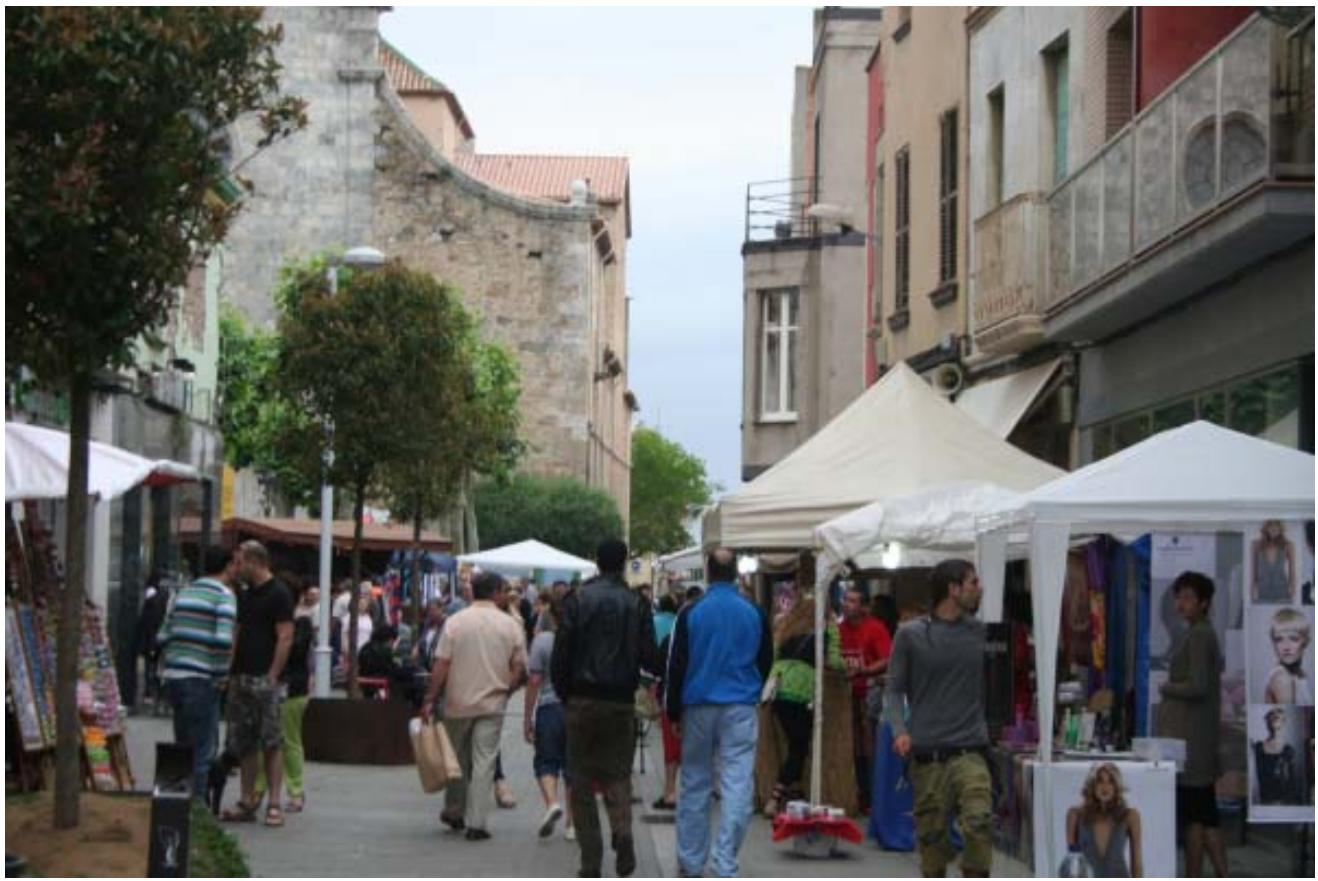

Fuente: Malgrat Comunicación (www.malgratcomunicacio.cat)

Finalmente remarcar que los comerciantes reconocen el esfuerzo de la administración local al apuntar que el Ayuntamiento tiene predisposición e interés en recuperar el comercio minorista para el centro y ha dedicado esfuerzos a potenciar el asociacionismo entre ellos. A su vez, los comerciantes valoran positivamente la 
acción realizada por la asociación en materia de dinamización para preservar su negocio e identifican nuevas estrategias de dinamización.

"El trabajo de la asociación es bueno, pero deberían realizar reuniones entre todos los comerciantes adscritos y ofrecer cursos de formación con el objetivo de mejorar individualmente el atractivo de cada comercio"

(Comerciante 10).

\section{Reflexiones finales}

Como conclusión general se puede argumentar que la crisis económica se revela como el principal factor de cierre del comercio minorista en Malgrat de Mar directamente vinculado a la disminución del consumo en los hogares. Asimismo, parece jugar un peso importante en este proceso la competencia generada como consecuencia de la apertura de supermercados localizados especialmente en el perímetro del casco urbano, dedicados sobre todo a la alimentación y productos para el hogar, con un conjunto de características atractivas para el consumidor que incluyen una superficie mayor que la del comercio minorista, zonas de estacionamiento propias y precios más competitivos que éstos últimos. También se apunta a los cambios de hábitos de los consumidores, la falta de relevo generacional y a la competencia generada por las compras on-line.

Para reactivar el comercio minorista local en un contexto desfavorable, desde antes de la crisis la administración local optó por la peatonalización de las principales calles comerciales del centro histórico. Si bien, como se ha podido comprobar a partir del análisis realizado, el impacto de esta estrategia fue dispar, en conjunto se puede afirmar que ésta fue positiva, atenuando en algunos casos los efectos de un contexto adverso (crisis e implantación de supermercados) y logrando superarlo en otros. Así, se aprecia que las calles peatonalizadas antes de la crisis se han enfrentado a ella con cierto vigor. Las calles peatonalizadas una vez iniciada la crisis, sin embargo, han sufrido un mayor porcentaje de cierre de locales comerciales, lo que parece evidenciar que la peatonalización por sí sola no ha sido suficiente para revitalizar el comercio local tradicional en este sector. Sin embargo, también es necesario puntualizar que en las zonas del centro peatonalizadas el comercio minorista ha resistido mucho mejor los efectos de la crisis que los establecimientos de servicios no especializados.

Los datos extraídos del análisis cualitativo realizado a actores principales del proceso, el Ayuntamiento y los comerciantes, amplían el análisis. Ambos colectivos convergen en señalar tanto la crisis económica como la implantación de supermercados en el entorno del municipio como las causas principales del declive del comercio local. A éstos, de todos modos, los entrevistados apuntan que se debe añadir otros factores como la falta de relevo generacional o la falta de adaptación a los nuevos hábitos de compra y consumo de los ciudadanos. En los aspectos positivos ambos colectivos coinciden en destacar la asociación de comerciantes como un factor significativo en la dinámica de ajuste y capacidad de respuesta del comercio local. Otros 
factores positivos identificados son la adaptación y especialización de los comercios a los nuevos hábitos de consumo.

\section{Bibliografía}

Atkinson, R. (2003): Domestication by cappuccino or a revenge on urban space? Control and empowerment in the management of public spaces, Urban Studies, 40(9), 1829-1843.

Ayuntamiento de Granollers (2013). http://www.granollers.cat/noticies/ajuntament/un-mapaciutat-mostra-cens-botigues-i-restaurants-web-granollers-mercat.

Ayuntamiento de Sant Cugat del Vallès (2014): Sant Cugat. Observatori sociològic, 2013. http://www.santcugat.cat/web/xifres.

Baringo, D., De Gaviria, M. (2001): Pequeño comercio y vitalidad urbana en Zaragoza. La ciudad contra la anti-ciudad. Revista Catalana de Sociología, 14, 185-190.

Batallé, D. (2000): Dels mercats i les botigues a les grans superfícies, Revista de Girona, 200, 97-106.

Bergua, E. (2002): Movilidad sostenible y comercio, Boletín CF+S, 19, 1-4. http://polired.upm.es/index.php/boletincfs/article/view/2597/2663

Borraz, F.; Dubra, J.; Ferrés, D.; Zipitría L. (2014): Supermarket Entry and the Survival of Small Stores, Review of Industrial Organization, 44, 1-73.

Campesinos, J.A. (1990): Centro-ciudad y revitalización funcional: Las calles peatonales cacereñas de Pintores y Moret, Eria, 22, 139-156. ISSN 0211-0563.

Casellas, A. 2016. "Desarrollo urbano, coaliciones de poder y participación ciudadana en Barcelona: una narrativa desde la geografía crítica" Boletín de la Asociación de Geógrafos Españoles, n. 70, 57-75.

Casellas, A., Dot, E., Pallares-Barbera M. 2010. “Creación de Imagen, Visibilidad y Turismo como Estrategias de Crecimiento Económico de la Ciudad” Finisterra. Revista Portuguesa de Geografía Vol. XLV, n. 90, 153-172.

Concello de Pontevedra (2011): Caminar resuelve: Peatones, seguridad y movilidad urbana. Hacia una inversión del esquema dominante. II Encuentro de Ciudades por la Seguridad Vial. Córdoba, 1-20. http://www.magrama.gob.es/es/ceneam/grupos-de-trabajo-yseminarios/movilidadinfantil/caminar-resuelve_tcm7-305097.pdf.

Conride, O. (2003 May 8): Sin comercio no hay ciudad, Confederación Argentina de la Mediana Empresa. http://www.redcame.org.ar/contenidos/noticia/Sin-Comercio-No-HayCiudad.430.html.

Cuesta, P. (1999): Centros comerciales en España. Concepto, tipología y evolución. Universidad de Alcalá. Distribución y consumo, 48, 1-17. ISSN 1132-0176.

Del Campo A., García, F.A., Flores, M. (2009): Proceso de peatonalización y nueva sociabilidad. Los casos de Sevilla y Málaga. Fundación Centro de Estudios Andaluces. 1144.

Espinosa Seguí, A. I. (2003): Amenazas y nuevas estrategias del comercio de centro urbano.

El caso de Alicante. Boletín de la Asociación de Geógrafos Españoles, 38, 153-174

Estudi de Mobilitat Obligada de Malgrat de Mar (2001):

http://xarxamobal.diba.cat/XGMSV/cat/municipis/municipis_indicadors.asp?codi_ine $=08110$. 
Fernandez, David (2009 Nov 21): La tienda y la amabilidad echan el cierre. Reportaje diario. El País. http://elpais.com/diario/2009/11/21/sociedad/1258758001_850215.html.

Franklin, A. W. (2001): The Impact of Wal-Mart Supercenters on Supermarket Concentration in U.S. Metropolitan Areas, Agribusiness, 17,105-14.

Goméz, R. (2000): El papel del comercio en la revitalización de los centros urbanos en Europa: las declaraciones de Málaga y Lille. Gestión y Análisis de Políticas Públicas, 19,71-78. ISSN 1134-6035.

González, M.J. (2006): La sostenibilidad de los centros históricos en los albores del siglo XXI. Anales de la Geografía, 26, 49-63.ISSN 0211-9803.

Herrera, N. (2012): Peatonalización y dinamismo comercial: El caso del centro histórico de la ciudad de La Laguna (Canarias-España). Proyecto de Investigación. Ciudad y calidad de vida. El uso social de los espacios públicos abiertos en las ciudades españolas, XX Coloquio de Historia Canario-Americana,107-117.

Instituto de Estadística de Cataluña (IDESCAT) (2017): www.idescat.cat.

Instituto Cartográfico y Geológico de Cataluña (ICC): www.icgc.cat.

Jacobs, J. (1961): Muerte y vida de las grandes ciudades. Madrid, Capitan Swing.

Matás, D.A (2013): El comercio en el barrio del Carmen. Contraclave, II Congreso sobre la identidad de la Región de Murcia, 1-10.

Pla Local de Seguretat Viària de Malgrat de Mar (2007-2010): http://transit.gencat.cat/web/.content/articles/arxius_ponencies_v_congres/jose_manuel_m artinez_experienciade_malgrat_de_mar.pdf.

Programa d'Orientació per als Equipaments Comercials de Malgrat de Mar (2009): https://www.ajmalgrat.cat/recursos-compartits/arxius/ajuntamentseuelectronica/información oficial/urbanisme/poec/text-refos.pdf.

Sanz, A. (1998): Elogio y censura de la peatonalización de los centros históricos. Ciudad histórica y calidad de vida, Burgos. Servicio de Publicaciones de la Universidad de Burgos y Fundación La Caixa.

Somoza-Medina, X. y López-González, A. (2017): Crisis, Comercio y Ciudad. Cambios en la Actividad Comercial y el Consumo en España. Revista de Estudios Andaluces, vol. 34 (1), 293-32.

Troitiño, M.A. (2003): La protección, recuperación y revitalización funcional de los centros históricos. Colección del Mediterráneo Económico. Ciudades, arquitectura y espacio urbano, 3, 131-160. ISSN 1698-3726.

Valbonesi, F. (2005): Malgrat de Mar (1975-2002). Creixement urbà dins de la Regió Metropolitana de Barcelona. Malgrat de Mar, Ajuntament de Malgrat de Mar.

Varisco, C. (2005): Recursos Comerciales en centros urbanos. Centro de Investigaciones Turísticas. Facultad de ciencias económicas y sociales de la Universidad de Mar del Plata, Aportes y transferencias, (9) 2, 112-126. ISSN 0329-2045.

Zukin, S. (1995): The Culture of Cities. Cambridge, MA: Blackwell.

Zukin, S. (1998): Urban lifestyles: Diversity and standarisation in spaces of consumption. Urban Studies, 35, 5-6, 825-839. 